\title{
Heinrich Hartmann
}

\section{Zwischen Projektionsfläche und Handlungsraum}

\author{
Raumvorstellungen bei Bayer und PCAC, I890 bis 1914
}

\section{ABSTRACT:}

Between projection screen and area of action Space conceptions at Bayer and PCAC, 1890-1914

The following paper seeks to conceive space as a core category of corporate organization, especially in periods of structural change. Based on two case studies from French and German chemical enterprises between 1890 and 1914 , it highlights how different agents tried to shape space in order to make it functional within the organisation of their enter- prises. The German Bayer company tried to actively define new borders and hierarchies inside the enterprise by planning a new plant at Leverkusen. By contrast, French PCAC's inorganic production was largely dependent on its natural environment in the Alps and at its sites near the Mediterranean Sea. Instead of actively transforming its organization, it tried to adjust it to its external determinants. In either case, space was a contentious factor in designing new corporate structures, whether treated as an external or a functional factor.

$\mathrm{E}$ iner der ersten Schritte in der Planung und Organisation eines Unternehmens besteht im Aufbau des Werksgeländes und des Unternehmensraums. Obgleich dies also chronologisch einer der ersten Managementakte darstellt, ${ }^{t}$ hat die Unternehmensgeschichte ihn bislang weitgehend übergangen. Zwar sind in letzter Zeit vermehrt neue organisationssoziologische Fragestellungen in die historische Analyse der Unternehmen einbezogen worden, doch gerade die räumliche Dimension, die in anderen wirtschafts- und sozialwissenschaftlichen Disziplinen eine wichtige Rolle zu spielen beginnt, ${ }^{2}$ blieb dabei in der Unternehmensgeschichte häufig unbeachtet. ${ }^{3}$ Fragen der

I Kornberger und Clegg sprechen von einer "tacit implication in mainstream organization theory», Martin Kornberger/Stewart Clegg, Bringing Space Back in. Organizing the Generative Building, in: Organization Studies 7 (2004), 1.095-I.I I 4; Franck Auriac, Système économique et espace. Un exemple en Languedoc, Université de Montpellier (unveröffentlichte Dissertation) 1979, 40; Yves Cohen, Organiser à l'aube du Taylorisme. La pratique d'Ernest Mattern chez Pengeot 1906-1919 (=Annales littéraires de l'Université Franche-Comté, Bd. 725), Besançon 200I, 14.

2 Stewart Clegg (Hrsg.), Space, Organizations, and Management Theory, Malmö 2006; André Spicer, Beyond the Convergence-Divergence Debate: The Role of Spatial Scales in Transforming Organizational Logic, in: Organization Studies Io (2006), 1.467-1.483; Tor Hernes, The Spatial Construction of Organization, Amsterdam 2004. Für die Geschichtswissenschaften sei in diesem Zusammenhang nur auf die entsprechende Thematik des Kieler Historikertages verwiesen Gerhard Fouquet/Arnd Reitemeier (Hrsg.), Kommunikation und Raum. Berichtsband des 45 . Historikertages in Kiel vom 14. bis zum 17. September, Neumünster 2005; auch David Gugerli/Daniel Speich, Topografien der Nation. Politik, kartografische Ordnung und Landschaft im 19. Jahrhundert, Zürich 2002.

3 Die einzige Ausnahme, die in Monografieform vorliegt, ist Stefan Blaschke, Unternehmen und Gemeinde. Das Bayenverk im Raum Leverkusen 1891-1914, Köln 1999. Hierzu auch der bald erscheinende Beitrag von Ruth Rosenberger auf dem 45. Historikertag in Kiel «Der Raum als Organisationselement;; Abstract in: Fouquet/Reitmeier, Kommunikation und Raum (wie Anm. 2), 43. 
regionalen Strukturforschung ${ }^{4}$ oder der Standortfaktoren ${ }^{5}$ sind historisch zwar durchaus in den Blick genommen worden, die internen Entwürfe und Pläne der Unternehmen spielten jedoch kaum eine Rolle. Dabei scheint die Frage nach Raumkonzeptionen und -vorstellungen der Unternehmen nicht nur das Verständnis der internen Führungsfunktion und der daraus hervorgehenden Zukunftsvorstellung $\mathrm{zu}$ fördern, sondern sie bietet auch Einblick in einen umstrittenen Organisationsbereich. In diesem verbinden sich unterschiedliche Vorstellungen, Konzeptionen und praktische Erfahrungs- und Wissensbestände miteinander und machen so die Konstruktion der Produktionsorganisation aus der Interaktion der Unternehmenszugehörigen heraus begreifbar. ${ }^{6}$ Als Hypothese ließe sich formulieren, dass sich der zugrunde liegende Organisationsaufbau eines Unternehmens in seiner Raumkonzeption niederschlägt und in Form von Werksplänen teilweise greifbar wird. Die Akteursgruppen, zwischen denen eine solche Raumkonzeption ausgehandelt wird, spiegeln daneben wider, auf welchen Ebenen Planungs- und Kontrollprozesse im Unternehmen angesiedelt werden.

Gleichzeitig werden durch Fragen der Organisation des Produktionsraumes auch divergierende Produktionspraktiken relevant. So darf räumliche Planung nicht isoliert von ihrer Umsetzung wahrgenommen werden. Erst durch technische Entwicklungen und soziale Dynamiken im Aufbau von Großproduktionen werden weitere konzeptionelle Überlegungen angestoßen und gewinnen als Managementinstrument an Bedeutung. Die Frage, welche Teile einer spezifischen lokalen Umwelt beispielsweise als Teil des Unternehmens definiert werden und welche nicht, zeigt sich als ein interaktiver Aushandlungsprozess, der die wechselseitigen Einflüsse zwischen Unternehmen und lokaler Umwelt in der historischen Analyse hervortreten lässt.?

4 Pat Hudson (Hrsg.), Regions and Industries. A Perspective on the Industrial Revolution in Britain, Cambridge u.a. 1989 .

5 Die lange zurückreichende Tradition der Standortfaktoranalyse basiert auf Alfred Weber, Über den Standort der Industrien. Teil 1: Reine Theorie des Standorts. Mit einem mathematischen Anhang von Georg Pick, Tübingen 1909. In der Wirtschaftsgeschichte wurde dieser Themenkomplexen immer wieder bearbeitet, besonders in Hinblick auf die Faktoranlayse der Protoindustrialisierung, etwa Hubert Kiesewetter, Das einzigartige Europa. Zufallige und notwendige Faktoren der Industrialisierung, Göttingen 1996; Stefan Gorißen, Vom Handelshaus zum Unternehmen. Sozialgeschichte der Firma Harkort im Zeitalter der Protoindustrie (1720-1820), Göttingen 2002, 48ff.; Karl Ditt/Sidney Pollard (Hrsg.), Von der Heimarbeit in die Fabrik. Industrialisierung und Arbeiterschaft in Leinen- und Baumwollregionen Westeuropas während des 18. und 19.Jahrhunderts, Paderborn 1992.

6 John B. Harley, The New Nature of Maps. Essays in the History of Cartography, Baltimore 200I, I53ff.; Hans-Dieter Schultz, Im Norden liegt..., nach Osten fließt... Vom Lesenlernen des Kartenbildes, in: Chrisof Dipper/Ute Schneider (Hrsg.), Kartenwelten. Der Raum und seine Repräsentation in der Neuzeit, Darmstadt 2006, 42-77; in allgemeinerer Perspektive Thomas Welskopp, Der Mensch und die Verhältnisse. ¿Handeln〉 und (Struktur) bei Max Weber und Anthony Giddens, in: ders./ Thomas Mergel (Hrsg.), Geschichte zwischen Kultur und Gesellschaft. Beiträge zur Theoriedebatte, München 1997, 39-70, hier s6ff.

7 Zur Bedeutung dieser wechselseitigen Austauschpunkte Hartmut Berghoff, Unternehmensgeschichte als Gesellschaftsgeschichte. Konzeptionelle Grundsatzüberlegungen am Beispiel des Aufstiegs Hohners vom dörfichen Heimgewerbe zum kleinstädtischen Großunternehmen, in: Jan-Otmar Hesse u.a. (Hrsg.), Kulturalismus, Neue Institutionenökonomik oder Theorienvielfalt. Eine Zwischenbilanz der Unternehmensgeschichte, Essen 2002, 243-25I, hier 248; Anne Nieberding, Unternehmenskultur im Kaiserreich.J.M. Voith und die Farbenfabriken vorm. Friedr. Bayer $\&$ Co. München 2003. 
Die folgenden Seiten sollen diese Fragen aus der Perspektive zweier Unternehmen beleuchten, die im Zeitraum vor I9I4 vor einer Vielzahl struktureller Veränderungen standen, die eine Reflexion ihrer räumlichen Entwicklung und Anordnung notwendig machten. Sowohl die technische Diversifizierung als auch die Expansion älterer Produktbereiche bildeten dabei den wirtschaftlichen Hintergrund für ihre partiellen geografischen Neuordnungen. Die Frage nach Raumplanungen und Raumentwürfen in Momenten strukturellen Wandels und technischer Integration sowie nach Akteuren und Aushandlungsmomenten dieser Planungen sollen dabei gleichzeitig eine Möglichkeit zur Vergleichbarkeit zweier Unternehmen aufzeigen, die sich den technischen Produktionen selbst erheblich voneinander unterschieden. Es handelt sich dabei einerseits um die Farbenfabriken vorm. Friedr. Bayer $\&$ Co (im Folgenden nur Bayer), die mit der Expansion in das neu zu erschließende Werk von Leverkusen einen deutlichen Anlass zum Überdenken ihrer bisherigen Produktionsaufteilung hatten, und andererseits um die südfranzösische Société des Produits chimiques d'Alais et de la Camargue (PCAC), die durch die Integration der Aluminiumproduktion ihren chemischen Produktionszirkel schloss und gleichzeitig neue Produktionsstandorte bauen und eingliedern musste. ${ }^{8}$

Ein Organigramm bauen die Erschließung Leverkusens durch das Bayerwerk

Die dramatische Expansion der deutschen Teerfarbenindustrie durch die Erlangung einer Quasimonopolstellung im letzten Drittel des I9. Jahrhunderts ist häufig beschrieben worden.9 Für Bayer bedingte die Ausweitung der Produktion immer wieder eine weitgehende Neuordnung der Produktions- und Werksräume. Schon der Standort Elberfeld war Ergebnis der Expansion der Farbproduktion: Durch die schnelle Abfolge neuer Entdeckungen in der Teerfarbenproduktion und eine kontinuierliche Ausweitung der Produktpalette wurden ab I 866 die Gründungsstätten am ursprünglich ersten Firmensitz in Barmen-Rittershausen zu klein, und die Firma musste sich einen neuen Standort für die Produktion suchen. Dieser wurde im nahe gelegenen Elberfeld gefunden, wohin das Unternehmen bald mit fast der gesamten Produktion umzog. ${ }^{\text {10 }}$ I 878 folgte auch die Verwaltung, und Elberfeld wurde zum neuen Unternehmenssitz, Barmen dagegen zu einem eher unbedeutenden Nebenstandort.

Doch auch der Betriebsstandort von Elberfeld war begrenzt von den an beiden Seiten aufsteigenden Anhöhen des Bergischen Landes. Die Werksleitung sah sich gezwun-

8 Diese Studien entstanden auf Grundlage meiner Dissertation "Moderne Zeiten? Unternehmensorganisation in Frankreich und Deutschland, um 1890-1914", die im Juli 2006 an der Freien Universität Berlin und der Ecole des Hautes Etudes en Sciences Sociales Paris verteidigt worden ist.

9 Gottfried Plumpe, Die IG-Farbenindustrie AG: Wirtschaft, Technik und Politik 1904-1945, Berlin I990; Johann Peter Murmann, Knowledge and Competitive Advantage. The Coevolution of
Firms, Technology, and National Institutions, Cambridge 2003; Blaschke, Unternehmen und Gemeinde (wie Anm. 3); Werner Abelshauser u.a., Die BASF. Eine Unternehmensgeschichte, München 2002; Anna Elisabeth Schreier/ Manuela Wax, Chronik der Hoechst Aktiengesellschaft 1863-1988, Frankfurt a. M. I990.

Io Gottfried Plumpe/Heinz Schultheis/Erik Verg, Meilensteine. 125 Jahre Bayer 1863-1988, Leverkusen 1988, 5 Iff. 
gen, die Produktionen mehrgeschossig übereinander aufzubauen. Um I 890 ließ sich jedoch ein neuerlicher Umzug nicht mehr verhindern. Was in den I860er und $70 e r$ Jahren vorexerziert worden war, wiederholte sich nun etwa 20 Jahre später. War die Firma allerdings noch mit wenigen hundert Arbeitskräften nach Elberfeld gezogen, so waren es Anfang der goer Jahre schon 3.I55 Arbeiter, zusammen mit den Beamten also über 3.500 Werkszugehörige. ${ }^{\text {I }}$ Das Unternehmen stand kurz davor, die meisten anderen chemischen Werke Deutschlands in der Größe zu überholen. ${ }^{12}$

Diese Expansionsgeschichte Bayers beruhte indes nicht nur auf der Ausweitung der Farbenproduktion; zeitgleich erschloss sich das Unternehmen neue Produktionszweige, in erster Linie durch die pharmazeutische und die anorganische Produktionsabteilung. Gerade aufgrund der veränderten technischen Bedürfnisse für diese Produktionslinien - für die anorganische Produktion bedurfte es in der Regel komplexerer technischer Anlagen - waren neue Produktionsstätten und -anlagen notwendig geworden. ${ }^{13}$

Die Werksleitung wollte erneuten räumlichen Problemen vorbeugen, indem sie eine Werksfläche wählte, die genug Platz für zukünftige Ausweitungen ließ. Die Gelegenheit hierzu ergab sich im rheinischen Wiesdorf, wo aufgrund des Todes von Carl Leverkus dessen Farbenfabrik mit einem beträchtlichen noch ungenutzten Terrain zum Verkauf stand. ${ }^{14}$ Mit Ausnahme des Flusses gab es hier zunächst keinerlei Restriktionen für eine weitere Expansion. Durch die Ansiedlung in Leverkusen musste allerdings endgültig auf das Prinzip räumlicher Geschlossenheit zwischen den einzelnen Produktionsstandorten verzichtet werden. Die Entfernung von knapp so Kilometern zwischen dem bergischen Elberfeld und dem neuen Ort, der bald den Namen Leverkusen erhielt, war zwar von der Post in einigen Stunden problemlos zu überbrücken, erlaubte den Direktoren aber keine kontinuierliche Präsenz an beiden Standorten.

Im Rückblick wurde der Akt der Erschließung des neuen Geländes vom Werk häufig glorifiziert, und diese "Pionierleistung" gehört bis heute zur Selbstdarstellung des Unternehmens. ${ }^{15}$ Doch entgegen diesem entschlossenen Bild verlief der Aufbau der Produktionsstätten in den ersten Jahren nach dem Ankauf derart zögerlich, dass ein genauerer Blick auf die Motivationen und Dynamiken innerhalb der Unternehmensleitung sinnvoll erscheint.

I I Allerdings zogen hiervon in der ersten Phase nur knapp 800 in das neue Werk um, Bayer Unternehmensarchiv (BAL) 265-5.3.

I 2 Nur die Ludwigshafener BASF war in Umsatz und Arbeiterzahl noch größer, dagegen war der Konkurrent Meister Lucius in Höchst bei Frankfurt bis zum Krieg überholt worden.

I 3 Vertraulicher Direktionsentwurf «Abtheilung für Anorganischen Großbetrieb", I 894, in: BAI IO-IS.

I4 Hans Joachim Flechtner, Carl Duisberg. Eine Biographie, Düsseldorf ${ }^{2} 1981$, 1366 .

Is Das Bild der Erschließung einer bis dahin "kaum bewohnten Gegend" kehrt in den Selbstdarstellungen des Unternehmens und später auch Carl Duisbergs immer wieder.
Dieser "Wild-West»-Topos ist grundlegend für den Mythos, den Leverkusen und dessen Aufbau noch heute im Unternehmen haben, aber auch in der Selbstinszenierung von Carl Duisberg als Chef des Unternehmens und Gestalter von Leverkusen. Er sprach etwa von "Mein[em] geliebte[n] Leverkusen, wie ich es mitschaffen durfte, und wie es heute als eine Musterstätte der chemischen Industrie dasteht"; Dankesrede Duisbergs zu seinem 25-jährigen Dienstjubiläum am 2.10.1909, zu dem ihm ein Bronzerelief Leverkusens geschenkt wurde, Carl Duisberg, Abhandlungen, Vorträge und Reden aus den Jahren 1882-1921, Berlin/Leipzig I923, 4I I; auch Carl Duisberg, Lebenserinnerungen, Leipzig 1933, 21. 
In den ersten vier Jahren nach Erwerb der Fabrik von Leverkus blieb das Gelände kaum genutzt, und erst langsam wurde die freie Fläche bebaut. Dabei kam in erster Linie den technischen Bauabteilungen eine entscheidende Bedeutung zu. Die Planung wurde zunächst von Technikern übernommen, die nicht direkt in die Produktion eingebunden waren. Hieraus begründete sich die wichtige hierarchische Stellung, die die Ingenieursabteilungen und die Betriebsingenieure für die Planungs- und Kontrollfunktionen bei Bayer einnahmen und die noch lange über die reine Erschließungszeit selbst für die Hierarchien im Unternehmen charakteristisch blieb. Während sich in der chemischen Produktion schon seit längerem eine zentralisierte Organisationsform durchgesetzt hatte, in der hierarchische Kontrolle zunehmend durch zentrale Abteilungen übernommen wurde, ${ }^{16}$ erlangten die Ingenieure, aber auch die handwerklich geschulten Werksmeister in dieser Zeit eine Schlüsselrolle im technischen und organisatorischen Aufbau des Unternehmens. ${ }^{17}$

Eine ähnliche Autonomie bekamen all diejenigen Abteilungen, die in dieser frühen Phase bereits in Leverkusen angesiedelt worden waren, allen voran die neue anorganische Abteilung. Sie unterstand mit ihrer Ansiedlung in Leverkusen nicht länger der Kontrolle durch das weiter in Elberfeld ansässige wissenschaftliche Laboratorium. Die beschäftigten Chemiker dieser Abteilung konnten nun autonom über die verwendeten anorganischen Zwischenprodukte und ihre Handhabung auf dem gesamten neuen Werksgelände entscheiden. Doch in den Produktflüssen zwischen Elberfeld und Leverkusen bekam die Abteilung beinahe den Rang eines externen Zulieferers, dessen Lieferungen wiederum genauso kontrolliert wurden, "als wenn sic von Concurrenzfabriken geliefert würden». ${ }^{18}$ Die räumliche Entfernung zwischen dem angestammten Unternehmenssitz in Elberfeld und den neuen Anlagen in Leverkusen stärkte zunächst die innerbetriebliche Tendenz zur Dezentralisierung von Entscheidungsbefugnissen und somit die Verlagerung von Hierarchie- und Kontrollstufen in die einzelnen Abteilungen.

Gegen diesen langsamen und von spontanen Erfordernissen geprägten Ausbau des neuen Werksgeländes wendete sich der junge Leiter des wissenschaftlichen Laboratoriums Carl Duisberg, der später noch die Meinung vertrat: "Man hätte besser getan, alles abzureißen und vollkommen neue Gebäude zu bauen.» ${ }^{19}$ Duisberg schrieb zum Jahreswechsel I 894/95 selbst einen Gegenentwurf. Mit dem Erscheinen dieser «Denkschrift über den Aufbau und die Organisation der Farbenfabriken zu Leverkusen» und dem Beschluss des Direktoriums, Duisbergs Plan weitgehend zu übernehmen, änderte sich die bisherige Situation schlagartig. ${ }^{20}$ Duisberg hatte diesen Entwurf in einer vorhergehenden Krankheitspause verfasst. ${ }^{21}$ Geschickt nutzte er somit die Leerstelle, die

I6 Ulrich Marsch, Zwischen Wissenschaft und Wirtschaft. Industrieforschung in Deutschland und Großbritannien 1880-1936, Paderborn u.a. $2000,40$.

17 «Pflichten und Obliegenheiten des Oberingenieurs und der Betriebs- und Werkstattingenieure", 1.I 1.1894, in: BAL IO-I5.

I 8 Vertraulicher Direktionsentwurf "Abtheilung für Anorganischen Großbetrieb", I 894, in: BAL IO-IS.
I9 Carl Duisberg. Ein deutscher Industrieller. Festgabe des Reichsverbandes der deutschen Industrie zum 29. September 1931, Berlin 193 I, 17.

20 BAL 003-004-OOI.

2 I Flechtner, Carl Duisberg (wie Anm. 14); Blaschke, Unternehmen und Gemeinde (wie Anm. 3). 
nach dem Aufkauf des Werkes entstanden war, um seinen eigenen Planungsentwurf für das Werk durchzusetzen. Er zeigte hierbei nicht nurVerständnis für die damaligen technischen Notwendigkeiten der Produktion, sondern formulierte auch explizit einen Plan für die künftige Entwicklung des Unternehmens:

"Wie eine Stadtverwaltung für Jahrzehnte hinaus Straßenlinien festlegt und Vorschriften für den Bau von Straßen, Kanälen, Gebäuden usw. erläßt, von denen nur ausnahmsweise abgewichen werden darf, so müssen auch wir heute schon alle unser Terrain später einmal durchschneidenden Hauptverkehrsstraßen genau festlegen, die dann selbstverständlich den wachsenden Bedürfnissen entsprechend erst nach und nach auszubauen sind." ${ }^{22}$

Das Tableau, das Duisberg in wenigen Winterwochen auf diese Weise ausmalte, sollte fortan beispielhaft für die Formulierung neuer räumlicher Entwürfe werden. Sowohl die Organisation der neuen Ingenieur-Abteilung und des für Bayer neuen Anorganischen Großbetriebs ${ }^{23}$ als auch die Umgliederung der technischen Verwaltung oder die Änderung der Geschäftsorganisation in Elberfeld ${ }^{24}$ gaben zukünftig Anlass zu umfangreichen Denkschriften, in denen versucht wurde, räumliche Gliederungen in ihrer Gesamtheit beschreibend zu erfassen und damit zu fixieren. ${ }^{25}$

Duisberg besaß zum Zeitpunkt seines Bauplanes für Leverkusen zwar die Prokura für das Unternehmen, gehörte selbst aber noch nicht dem Direktorium an. Durch diesen Entwurf stellte er zum ersten Mal seine Vision des Unternehmens und seiner zukünftigen Entwicklung vor und etablierte sich damit dauerhaft in der Rolle des "Organisators". Neben den Direktoriumsmitgliedern war auch er berechtigt, die technischen Entwürfe für zukünftige neue Betriebsanlagen gegenzuzeichnen und damit den Aufbau des Werkes in seiner Realisierungsphase entscheidend $\mathrm{zu}$ beeinflussen. ${ }^{26}$ Die Schrift legte teilweise die Grundlage für seinen späteren Aufstieg im Unternehmen, der mit der Ernennung zum alleinigen Generaldirektor des Unternehmens im Jahr I9I2 ihren Höhepunkt erreichte. Der räumliche Entwurf für Leverkusen nahm somit einen entscheidenden Platz in der Entwicklung von Duisbergs persönlicher Karriere ein.

Die Duisberg'sche Konzeption spielte eine entscheidende Rolle bei der weiteren Entwicklung von Bayer. Sie stützte sich dabei auf drei grundlegende Prinzipien, die den Raum zu einer Kernkategorie nicht nur in der technischen, sondern auch in der sozialen Konstruktion der Produktionsorganisation werden ließ: I. Der Raum wurde austauschbar gemacht und in gleich große Parzellen zerlegt, die dann den einzelnen Produktionsprozessen und Betrieben zugeteilt waren. Bei einem dauerhaft gesteigerten Bedarf an einem Produkt wurde künftig nicht mehr der Betrieb so lange vergrößert,

22 BAL $003-004-001$.

23 Organisation der Ingenieur-Abteilung zu Leverkusen, I0.10.1900; Abteilung für Anorganischen Grossbetrieb, ohne Datum [1894], in: BAL IO-Is.

24 Organisation der technischen Verwaltung der Farbenfabriken vorm. Friedr. Bayer \& Co, ohne Datum [um I905]; Denkschrift über die Organisation des Gesamtgeschäfts der
Farbenfabriken vorm. Friedr. Bayer \& Co zu Elberfeld, Februar I906, in: BAL I0-3-I.

25 Von entscheidender Bedeutung zum Verständnis ihres Anspruchs ist die narrative Form, in der diese Denkschriften abgehandelt wurden, in: BAL I0-3-I.

26 "An die Herren Betriebsführer in der Filiale der Farbenfabriken vorm. Friedr. Bayer Co. Leverkusen", in: BAL I2-I8. 
bis ihm der Platz fehlte, vielmehr wurde ein zweiter Betrieb für das gleiche Produkt eröffnet. Hieraus ergab sich eine Standardisierung der Abteilungs- und Betriebsgrößen. 2. Die Produktionsbetriebe mussten zu diesem Zweck geteilt werden. Die Versorgungsbetriebe, die nur Zwischenprodukte herstellten oder die mit der Anlieferung betraut waren, bekamen eine zentrale, für alle anderen Betriebe erreichbare Lage. Hiermit wurde also eine Hierarchie der einzelnen Abteilungen untereinander festgeschrieben. Dies war ebenso der Fall für die Trennung der Betriebe zwischen Elberfeld und Leverkusen. Auf das neue Gelände in Leverkusen kamen in erster Linie die jungen zukunftsträchtigen Abteilungen mit der Produktion von Endprodukten. Dagegen blieben in Elberfeld zunächst die Verwaltung und das zentrale wissenschaftliche Labor. Über die technischen Neuerungen, die starke Expansion und Angliederung neuer Produktionszweige - etwa die Eröffnung der neuen Photographischen Abteilung ab 1912 - gewann das Leverkusener Werk schnell eine solche Prädominanz, dass 1912 die Verwaltung ebenfalls von Elberfeld nach Leverkusen wechselte. 3. Mit diesem Schritt wurden nun die Arbeiter auf einen räumlich eng umgrenzten Arbeitsplatz festgelegt. Die Arbeitsverträge, die im Folgenden bei Bayer abgeschlossen wurden, bestimmten, dass es dem Beschäftigten nur noch erlaubt war, "diejenigen Fabrik-, Bureau, Comptoirs, Lagerund sonstigen Arbeitsräume zu betreten, welche ihm für seine Thätigkeit angewiesen sind.$^{27}$ Der Zugang für nicht dem Werk Zugehörige war vollständig ausgeschlossen. Dieser Grundsatz hatte direkte Konsequenzen auf die aktive Arbeitseinteilung, die auf dieser Weise einer zentralen Kontrolle unterworfen war.

Doch nicht nur bei den Produktionsabteilungen selbst, sondern auch bei der jeweiligen Betriebsleitung griff Duisbergs Plan in die räumliche Ordnung ein. ${ }^{28}$ Ergänzend zu seiner Denkschrift für Leverkusen lieferte er einen Plan für die Verwaltungsgebäude der einzelnen Abteilungen, in denen das Prinzip räumlicher Kontrollierbarkeit reproduziert wurde. Der Plan dieser wissenschaftlichen Laborräume war das einzige bildliche Dokument, das Duisberg seiner Denkschrift hinzufügte und mit dem er definierte, wie das organisatorische Herz der einzelnen Abteilungen auszusehen hatte. Allen Betriebschemikern einer Abteilung wurden zusammen zwei Schreibzimmer zugewiesen, ihr Hauptarbeitsbereich befand sich an den Experimentierplätzen. Einen verhältnismäßig großen Raum nahm die Bibliothek ein.

Der Raum sollte von der Direktion einsehbar sein, die verschiedenen Abteilungen zu jedem Zeitpunkt von der Direktion kontrolliert werden können. Das Prinzip von Konstruktion und Klassifikation des Raumes wurde in der Folgezeit und spätestens mit dem Eintritt von Duisberg ins Direktorium zum allgemeinen Prinzip erhoben. 1903 wies er die Betriebsführer an, bei allen weiteren Vorgängen seinem Beispiel zu folgen:

«Soll ein Betrieb von Elberfeld nach Leverkusen verlegt, oder dort ein neuer Betrieb errichtet oder ein solcher vergrössert oder umgebaut werden, so hat der-

27 Vertragsvordruck von 1903 , in: BAL 213.1 . $28 \mathrm{Er}$ kopierte hierfür einen Grundsatz, den er schon im von ihm geleiteten Zentrallabor angewendet hatte: "Während es bis dahin in der chemischen Industrie eine ererbte Regel war, dass jeder Chemiker einen durch
Wände und Türen abgegrenzten Raum hatte, führte er das Boxsystem ein, so dass alle wie in einem Universitätslaboratorium ohne geistige und räumliche Schranken beieinander waren.», in: Alfred Stock, Carl Duisberg, 29.9.1861-19.3.1935, Berlin 1935, 12. 
Planvorlage für die Verwaltung der Abteilungen, 1895

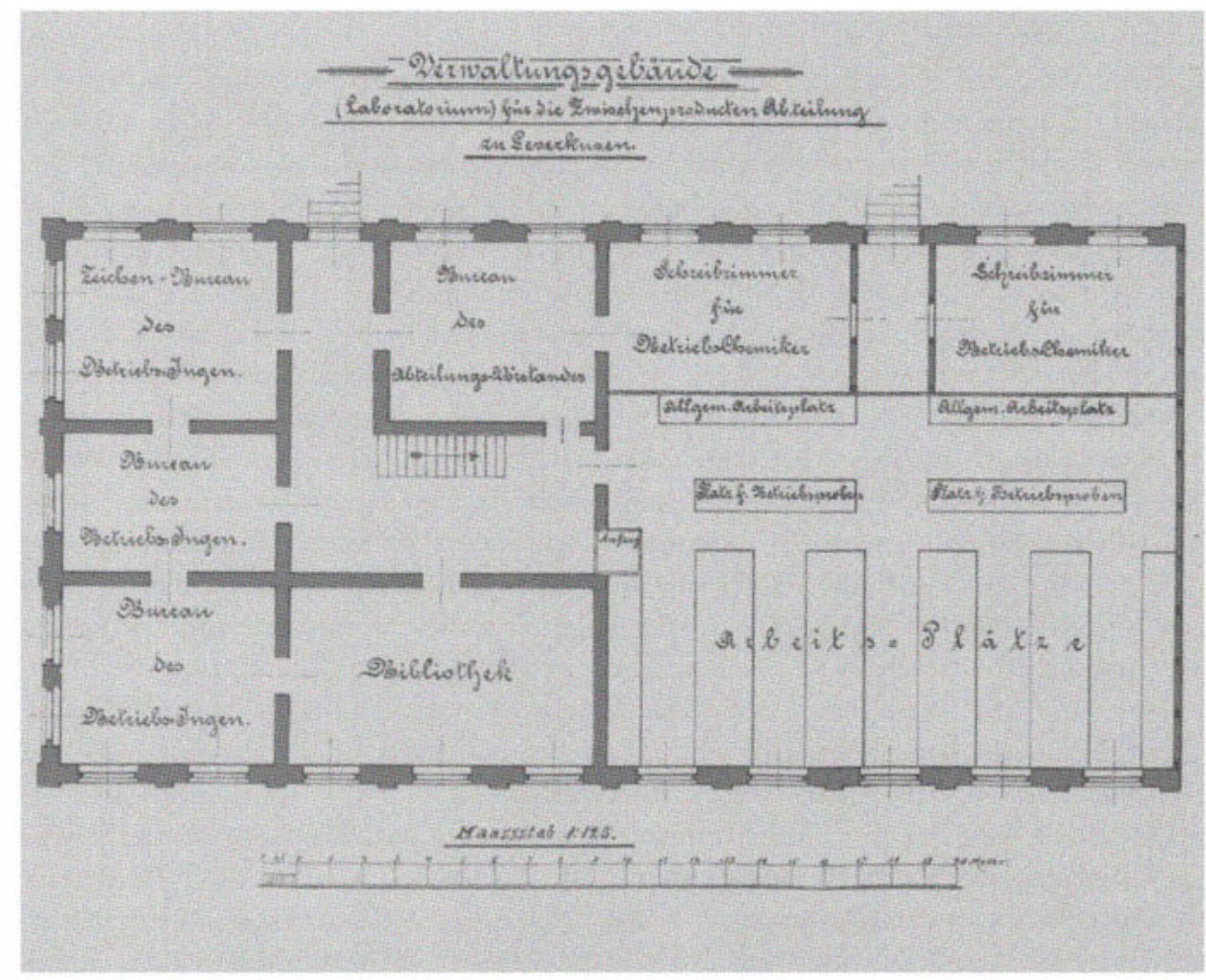

Quelle: BAL 003-004-001, Anhang.

jenige Abteilungsvorstand und derjenige Betriebschemiker, der mit der Verlegung bzw. Errichtung oder Vergrösserung des Betriebes und der späteren Betriebsführung betraut wird, zuerst eine allgemein gehaltene Beschreibung der gesamten Operationsfolge unter Angabe der Art und Folge der Apparate, nebst einer Handskizze anzufertigen und diese dann in einer Besprechung mit dem Vorsteher des Konstruktionsbureaus in Gegenwart des mit der Ausführung der Konstruktionsarbeit $\mathrm{zu}$ betrauenden Ingenieurs zu erläutern. ${ }^{29}$

Die organische Chemieindustrie war in Deutschland weit weniger von räumlichen Determinanten geprägt als viele andere Industriezweige. Die Frage, wo sich ein chemisches Unternehmen ansiedelte, war in geringerem Maße von Standortfaktoren abhängig. Zwar stellte die Versorgung mit Rohstoffen eine Notwendigkeit dar, sie war allerdings quantitativ und qualitativ weniger von einer bestimmten Quelle dieser Rohstoffe abhängig als beispielsweise der Bergbau oder die Stahlindustrie des Ruhrgebietes.

29 Rundschreiben von Carl Duisberg an alle Chemiker und Ingenieure vom 31.7.1903, in: BAL 210. 
Damit waren Handlungsspielräume, wie sie Duisberg in der Planung der Topographie des Unternehmens nutzte, umso größer.

Der von Duisberg verfasste Plan versuchte, die bislang spontane Bebauung des Geländes in Leverkusen zu systematisieren. Es gab keine anderen schriftlich fixierten Überlegungen. Doch der von Duisberg entwickelte Plan und seine bauliche Umsetzung erfassten nicht die reale Ordnung des Unternehmens, sodass eine Vielzahl von Korrekturmaßnahmen nötig wurde, die die Werksleitung in den kommenden Jahren vornahm. Erst durch diese Änderungen setzte die Werksleitung ihre eigenen Vorstellungen eines zentralisierten Betriebszusammenhanges durch.

Schon das Prinzip der Abgrenzung von Werksinnerem und -äußerem und der damit verbundene Übergang von einer personalisiert visuellen zu einer distanzierten und systemischen Kontrolle des Produktionsraumes ${ }^{30}$ machte eine gewisse Anzahl neuer Kontrollinstitutionen notwendig. Den Zugang zum Werk kontrollierten Pförtner an den Werkstoren. ${ }^{3 \mathrm{r}}$ Mit der Erschließung Leverkusens wurde hier - wie zuvor in Elberfeld ein Fabrikkontor als zentrale Personalstelle eingerichtet. Hierdurch war arbeitssuchenden Bewerbern der Zutritt auf das Werksgelände untersagt. Sie erhielten ihn erst nach einer vorher ausgehandelten Anstellung. ${ }^{32}$ Die Zeit, in der Bewerber persönlich in den Abteilungen vorstellig wurden, war damit beendet.

Innerhalb des Werkes spielte die Werksfeuerwehr eine Schlüsselrolle, die durch ihre zentralen Befugnisse, wie etwa die Ermahnung und Entlassung von Arbeitern, beinahe polizeiliche Bedeutung erhielt. ${ }^{33}$ Zusätzlich zum baulichen und technischen Entwurf wurde das Werksgelände damit zu einem Raum, der auch über Machtstrukturen definiert war, die außerhalb des Werkes keine Bedeutung hatten. Sowohl das Vorhandensein einer solchen Form werksinterner Kontrolle als auch die Zunahme von Rundschreiben, die die Definition von Arbeitsbereichen zum Ziel hatten, sind Indikatoren für die langwierigen Aushandlungsprozesse zwischen Unternehmens- und Betriebsleitern einerseits, und der Belegschaft andererseits, die durch den Duisberg'schen Plan ausgelöst worden waren. ${ }^{34}$

Auch das Betriebsäußere wurde zunehmend Teil der unternehmerischen Planung. Durch die zahlreichen Wohlfahrtseinrichtungen und besonders aber durch den massiven Bau von Werksheimen und Werkswohnungen war das Unternehmen in der privaten Sphäre der Belegschaft präsent. ${ }^{3 s} \mathrm{Ob}$ allerdings die intendierte Wirkung eines solchen versuchten Eingriffs in die private Lebenssphäre der Werksangehörigen die

30 Jean-François Chanlat, Space, Organization and Management Thinking, in: Clegg u.a., Space (wie Anm. 2), 17-44, hier 18.

3 I «Handbuch für die Arbeiter der Farbenfabriken vorm. Friedr. Bayer \& Co.m, ohne Jahr [nach I900], 1 , in: BAL I0-8-2.

32 Rundschreiben ohne Datum [etwa Ig05], in: BAL 10-4.

33 "Den Anordnungen der Feuerwehrleute, sobald sie umgeschnallt haben, und der Pförtner, sobald sie in Uniform sind, ist in allen Fällen unbedingt und ohne Verzug Folge zu leisten,* Fabrikordnung von I 899 , in: BAL IO-IS.
34 Als ein Beispiel soll hier derVersuch der Werksleitung genannt werden, Pausen- und Arbeitsbereiche in den Betrieben räumlich so von einander zu trennen, dass es den Arbeitern nicht mehr möglich war, ihre Kaffeepausen mit ihrer Arbeitszeit zu mischen, "Ueber die Arbeitszeit der Arbeiter, in: BAL IO-I 5 .

35 Dieser Werkswohnungsbau ist umfassend dargestellt von Nieberding, Unternehmenskultur (wie Anm. 7), S. $167 f$. Hiernach stieg der Anteil der "Werkswohnen" an der Belegschaft von $10 \%$ I 897 auf über $20 \%$ vor Ausbruch des Ersten Weltkriegs und lag damit signifikant höher als in anderen Industrieunternehmen. 
gewünschten Erfolge - also die Stabilisierung der Arbeitskräfte - zeitigte, darf vor dem Hintergrund zahlreicher disziplinarischer Vorfälle bezweifelt werden. ${ }^{36}$

Die Übersiedlung der Produktion nach Leverkusen verweist auf einen Grundkonflikt in der Geschichte von Bayer vor I914: Die räumliche Distanz verstärkte die Spannungen zwischen den Ambitionen einer zentralen Unternehmensführung und der Autonomie einzelner Produktionsabteilungen. Dies galt umso mehr, als durch die technischen Notwendigkeiten der Umsiedlung die Betriebsleiter, Ingenieure sowie die Handwerker an Bedeutung gewannen. Für einzelne Abteilungen bedeutete der Umzug nach Leverkusen damit nicht allein eine Ausweitung ihrer Produktionsfläche. Der räumliche Abstand nach Elberfeld verschaffte ihnen auch Unabhängigkeit von der Zentralverwaltung. Der von Carl Duisberg verfasste Entwurf für das neue Werksgelände lässt sich zunächst als Zukunftsplan für die Entwicklung des Unternehmens lesen. Daneben stellt er jedoch den Versuch dar, eine zentrale Kontrolle aufrechtzuerhalten und diese auf dem neuen Werksgelände topografisch festzuschreiben. Doch auch wenn technologische Faktoren den Ausschlag für diese Planungsbemühungen gaben, so zogen sie die Notwendigkeit zur Reformulierung des sozialen Unternehmensraumes nach sich. Die Neuanordnung der Produktionsstätten machte somit eine Erfassung und Umschreibung des unternehmerischen Machtraumes notwendig.

Die räumliche Ordnung, die ein Unternehmen prägt, ist in entscheidendem Maße abhängig von seinen Produktionstechniken. Eine technische Umstrukturierung oder die Integration neuer technischer Verfahren bietet in diesem Zusammenhang auch die Möglichkeit zur Ausweitung von Handlungsräumen der einzelnen Abteilungen. Wie schon am Beispiel der anorganischen Abteilung bei Bayer gesehen, boten die Neuansiedlung in Leverkusen und die räumliche Entfernung von der Zentralverwaltung Anlass zur Neuordnung hierarchischer Gefüge, die wiederum das Gesamtunternehmen zur Reaktion zwangen.

\section{Die Ordnung des Raumes durch Kommunikation - PCAC}

In noch höherem Maße als in der organischen Chemie waren Unternehmen der anorganischen Branche von Zwängen, wie etwa Energieangebot und Rohstoffvorkommen, in ihrer räumlichen Organisation geprägt und mit ihrer Umwelt verbunden. Das südfranzösische PCAC, das anorganische Grundstoffe produzierte, diversifizierte seine Produktpalette ebenfalls in den drei Jahrzehnten vor dem Ersten Weltkrieg nachhaltig. $\mathrm{Zu}$ den landwirtschaftlich benötigten Produkten wie Kupfersulfat gesellten sich im letzten Jahrzehnt des I9.Jahrhunderts zunehmend Grundstoffe für die Aluminiumproduktion, vor allem Tonerde. Daneben expandierte das Unternehmen sowohl in den Bereich der hierzu benötigten Rohstoffgewinnung - neben dem Abbau von Kohle und Schwefel vor allem die Meersalzgewinnung - als auch ab 1897 in die

36 Nieberding führt hier etwa die zahlreichen disziplinarischen Probleme in den Junggesellen- und Mädchenheimen an und kommt zum Schluss: «Nutzen im Sinne von Versteti- gung bzw. Erziehung zu wirtschaftsfriedlichem Verhalten [...] erzielten diese Einrichtungen nicht.», Nieberding, Unternehmenskultur (wie Anm. 7), 184. 
elektrochemische Aluminiumproduktion selbst. ${ }^{37}$ Mit dieser Expansion integrierte PCAC den gesamten Produktionszyklus der Aluminiumherstellung; ein Schritt, den bereits einige direkte Konkurrenten gemacht hatten. ${ }^{38}$

Diese Diversifizierungsstrategie hatte zur Folge, dass das Unternehmen sich gerade im letzten Jahrzehnt des I9. Jahrhunderts auf weite Teile des französischen Territoriums ausweitete. Neben dem Stammwerk von Salindres in den Cevennen, das weiterhin einen Großteil der chemischen Grundstoffproduktion sicherstellte, gewannen diverse Rohstoffvorkommen, insbesondere die Salinenanlagen von Salin de Giraud in der Camargue, an Bedeutung. Hinzu kamen bis I9I4 drei Betriebe zur hydroelektronischen Aluminiumverhüttung im savoyardischen Tal der Maurienne. Dieser Aufschwung führte zu einem rasanten Anstieg der Arbeiterzahlen an den verschiedenen Unternehmensstandorten. ${ }^{39}$

Die Ausweitung der Produktion löste bei PCAC schwerwiegende Kontroll- und Koordinationsproblemen aus. Die einzelnen Betriebe nutzten die räumliche Unabhängigkeit von der Zentralverwaltung, um sich Entscheidungsspielräume, etwa in Bereichen der Tarifgestaltung oder der technologischen Entwicklung, zu sichern. Auch bei PCAC wurde damit das Wechselspiel zwischen zentralen und dezentralen Strukturen zu einer Kernproblematik der Zeit vor dem Ersten Weltkrieg. ${ }^{40}$ In diesem konstanten Aushandlungsprozess zwischen den Standorten und der Verwaltung griff PCAC vor dem Krieg allerdings noch nicht auf verschriftlichte Organisationsentwürfe oder Entwicklungspläne zurück. ${ }^{\text {I }}$ Vielmehr kristallisierte sich in dieser Zeit eine Verwaltungspraxis heraus, die stark auf kommunikativen und weitgehend personalisierten Strukturen beruhte. Sie sah eine Hierarchisierung zwischen den einzelnen Unternehmensstandorten vor, die dabei in ihrem Grundmuster ebenso fest gefügt war, wie das durch schriftliche Pläne der Fall gewesen wäre. ${ }^{42}$ So mahnten die Unternehmensleiter - bis 1907 Alfred Péchiney, anschließend Adrien Badin - die verschiedenen Unternehmensabteilungen immer wie-

37 Muriel Le Roux, L'entreprise et la recherche. Un siècle de recherche industrielle à Péchiney, Paris I998; Paul Toussaint, Historique de la Compagnie Pechiney, Manuskript, zugänglich über IHA Paris, I 950; Ludovic Cailluet, Stratégie et structures d'organisation et pratiques de gestion de Pechiney des années 1880 à 1971, Université Lyon II (unveröffentlichte Dissertation) I99S.

38 Florence Hachez-Leroy, L'Aluminium Français. L'invention d'un marché, 1911-1983, Paris 1999.

39 So werden in einer Untersuchung der Departementsverwaltung für das Unternehmen im Jahr I 890670 chemische Arbeiter, 60 Hilfsarbeiter, 14 Werksmeister und 17 surveillants genannt, insgesamt also 762 Beschäftigte, eine Zahl, die sich bis zum Krieg um $65 \%$ steigern sollte, in: Archives Départemetales du Gard, 9 M 40; Archives Crédit Lyonnais Etude économique et financière I91 3, 21.124.

40 Hiervon zeugen die immer wieder im Verwaltungsrat des Unternehmens besprochenen Vorschläge zur Umstrukturierung der Ver- waltung, die sich als ein ständiges Hin und Her zwischen Autonomie der Standorte und Zentralisierung der Verwaltung lesen lassen. Etwa Procès verbaux du conseil d'administration, 28.7. 1898 und 28. I I. I90I, in: Archiv des Institut pour l'histoire de l'aluminium, Abteilung Péchiney (Py) 072/13/29960.

4I Die Schriftensammlung zu Problemen der Organisation als eigenes Problemfeld beginnt im Jahr 1919, die ersten Organisationsentwürfe stammen erst aus den 20 er Jahren, in: Py oo/ro/10018.

42 Diese Kommunikationsstrukturen wurden von der Unternehmensleitung auch immer wieder explizit reflektiert, zum Beispiel bei der Eingliederung von neuen Unternehmensstandorten in das bestehende Unternehmensnetz (Brief vom 1.2.I 898, in: Py IHA 962502 Prim6/9) oder aus Anlass von Umstrukturierungsmaßnahmen, die in ihren Auswirkungen auf Kommunikationsstrukturen überdacht wurden (Brief von Boyoud an den Direktor Perry in Salin de Giraud am 2.12.1910, in: Py 00/12/20018). 
der, die ungeschriebenen Regeln des Unternehmens und damit dessen informelle Hierarchie einzuhalten. I9I 3 schrieb beispielsweise der Generalsekretär Paul Vittenet eine äußerst harsche Mahnung an die Aluminiumhütte von Calypso, adressierte diese aber nicht etwa an die Fabrik selbst, sondern an deren "vorgesetzte» Fabrik in St. Jean-enMaurienne. Er ergänzte diese Ausführungen mit dem handschriftlichen Hinweis: «Beim Schreiben des Vorangegangenen hatten wir im Sinn, dass Calypso immer St. Jean untergeordnet war. Und in der Realität schreiben wir an Calypso ausschließlich über den Umweg der Fabrik in St. Jean." ${ }^{43}$

In solchen Aushandlungsprozessen wurde wiederum die räumliche Distanz zwischen den Produktionsstandorten, die eine prinzipielle Komplikation der Kommunikationsstrukturen bedingte, zum schlagkräftigen Argument. Doch erst kurz vor dem Krieg formulierte die Unternehmensleitung explizit ihre Vorstellung vom dezentralen Unternehmen, dessen Zentralisierung man sich nun ausschließlich über die Kommunikation vorstellen konnte. Dieser Grundsatz spiegelte im Prinzip die bereits lange vorher gültige Organisationspraxis wider:

"Die Dezentralisierung der Handlungen, die wir so [durch die Autonomie der einzelnen Standorte] erhalten, muss von einer Zentralisierung der Informationen, die für die Unternehmensleitung notwendig sind, begleitet werden. Es ist somit Sache der verschiedenen Abteilungsleiter, den jeweiligen Stand ihrer Geschäfte regelmäßig in ihren Berichten und ihrem Schriftverkehr niederzulegen [Herv. i. Original]. $\rangle^{44}$

Anders jedoch als bei Bayer beließ die Unternehmensleitung es allerdings bei dieser Informationszentralisierung. Sie versuchte nicht, durch die Entwicklung von Werksplänen die einzelnen Standorte zentral zu organisieren. Gerade die Koordinationsfunktion der Ingenieure nahm in den folgenden Jahren in der Tendenz weiter zu. Hatte noch in den I 890er Jahren der Direktor des Unternehmens, Alfred Péchiney, versucht, technische Entscheidungen weitgehend selbst zu treffen, entwickelte sich bis $19{ }_{4} 4$ mit den Ingenieuren und Werksdirektoren eine gewichtige neue Hierarchieebene im Unternehmen.45

Die Werksgelände der einzelnen Produktionsstandorte wiesen eine geringe räumliche Geschlossenheit auf. Am Beispiel der neuen Produktionsstandorte für die Aluminiumverhüttung in den französischen Alpen wird dies in besonderem Maße augenfällig. Zwar wurde das Herz der Betriebe durch einzelne Gebäudeanlagen gebildet, allerdings weitete sich das Unternehmen im Tal weit über diesen Produktionskern hinaus aus. Die zur Gewinnung der hohen Energiemengen benötigten Wasserfallrohre und Talsperren, die den Anblick des ganzen Tals der Maurienne dominierten, bildeten einen integralen Bestandteil des Unternehmens. Nimmt man die frühe Werksfotografie dieser Zeit als Form der unternehmerischen Eigenrepräsentation, so belegen diese Fotos, dass das Unternehmen wie selbstverständlich große Teile des Tales als Teil seiner Pro-

43 Brief vom 19.11.1913, in: Py 00/12/20012. 44 Organisation des Services de la Compagnie, 5.5.1914, in: Py 00/12/20012. Diese einzige Schrift zur Organisation des Unternehmens als Ganzes wurde von Badin ausgearbeitet und war schon im Februar dem conseil d'Administration vorgestellt worden, Protokoll vom 6.2.19I4, in: $\mathrm{Py} \mathrm{072/13/29960.}$ 45 Cailluet, Stratégie et structures (wie Anm. 37). 


\section{Bauphase von Calypso mit Wasserrohrsystem 1897}

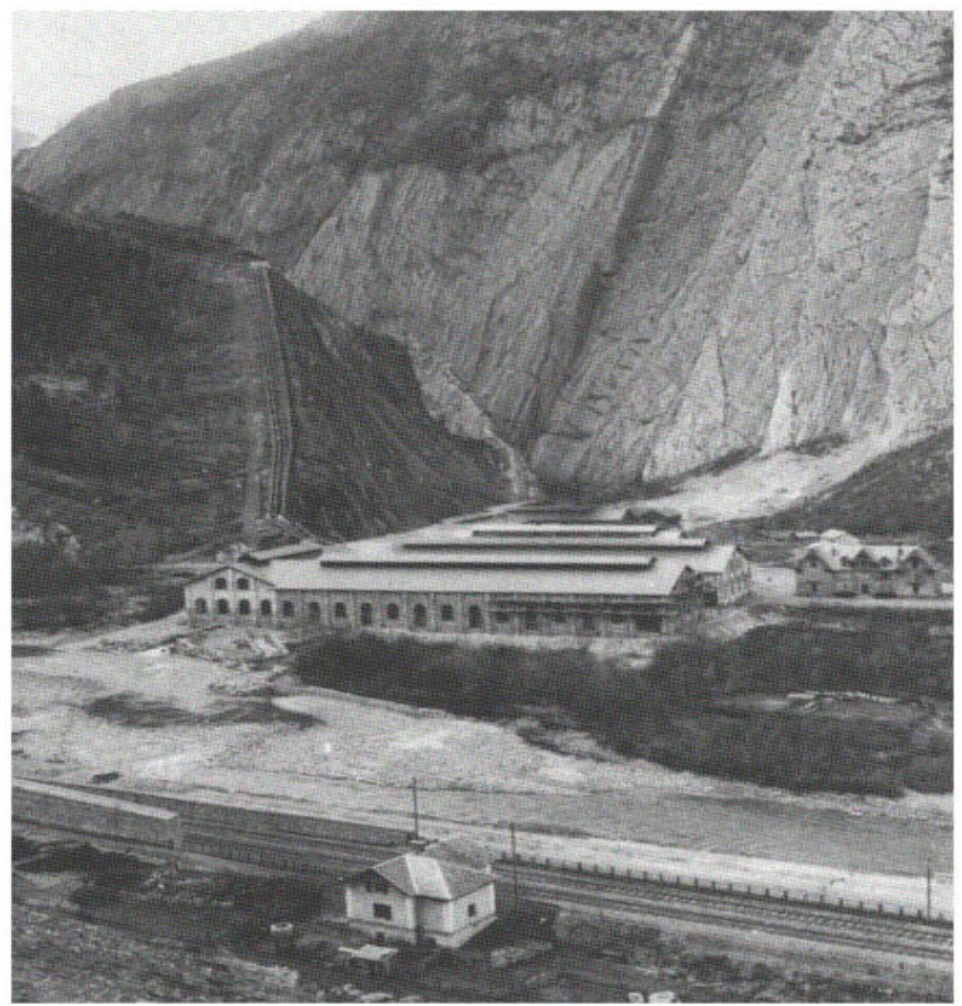

Quelle: Py 080/12/1994I.

duktionsstätten mit definierte. Nahezu alle diese Fotografien beziehen die technischen Anlagen und Fallrohre außerhalb des direkten Werksgeländes mit ein.

Eine ähnlich offene Topologie wiesen auch die älteren Produktionsstandorte auf. Nicht nur aufgrund des Energiebedarfs, auch zur Sicherstellung einer ausreichenden Wasserversorgung besaßen die Betriebe in Salindres und in Salin de Giraud ebenfalls dreimal so viel Platz, wie die eigentliche Produktionsfläche in Anspruch nahm..$^{46}$ Die Werksgelände waren somit einerseits weniger abgeschlossen als bei Bayer und griffen andererseits weit über die Produktionsstätten hinaus auf ihre ländliche Umwelt über.

Diese starke Abhängigkeit des Unternehmens von seiner direkten Umwelt wurde in verschiedenen Beziehungen sichtbar. Wie auch Bayer wurde PCAC schon früh für die Umweltbelastung seiner Produktion verklagt. ${ }^{47}$ Doch trotz offensichtlicher Verschmut-

46 Unternehmensbeschreibung von 1913 , in: Archives Credit Lyonnais, DEEF 21124.

47 Historique du Salindres [1919], in: Py $00 / 12 /$ 2001 I. Für Bayer ist dieses Problem inzwischen von mehreren Autoren bearbeitet worden. Blaschke, Untermehmen und Gemeinde (wie Anm. 3), 4off.; Ralf Henneking, Chemische Industrie und Umwelt. Konflikte und Umweltbelastungen am Beispiel der schwerchemischen Industrie, Farben- und Düngemittelindustrie in der Rheinprovinz, Stuttgart 1994. 
zungen von Feldern und Wäldern und der daraus entstehenden Ernteausfälle wurde das Unternehmen von offizieller Seite nicht belangt. ${ }^{8}$ Der "Verbrauch" des landwirtschaftlichen Raumes war ihm von dieser Seite zunächst weitgehend freigestellt.

In anderer Beziehung wurden dagegen die Abhängigkeiten für das Unternehmen deutlicher: PCAC stützte sich in seiner Organisation auf die Integration lokaler landwirtschaftlicher Strukturen. Ein Großteil der Arbeitskräfte waren Kleinbauern, die einen Hof und einige Felder hatten. ${ }^{49}$ Der Personalbedarf des Unternehmens musste sich unter anderem nach den Erntezeiten vor Ort richten, und erst durch dieses Wechselverhältnis konnte eine dauerhafte Bindung der Arbeitskräfte erreicht werden. Über die genaue Anzahl von Arbeitern bei PCAC gibt es daher auch wenig Auskunft, da viele von ihnen nicht durchgängig beschäftigt waren, sondern beispielsweise nur einige Tage im Monat für das Unternehmen arbeiteten. ${ }^{\text {so }}$ Diese starken Abhängigkeiten von der Landwirtschaft als Lieferant eines ausreichenden und stabilen Angebots an Arbeitskräften, aber auch als Abnehmer der chemischen Grundprodukte war somit inhärent. Landwirtschaftliche Konjunkturen hatten direkte Auswirkungen auf die Personalorganisation und bestimmten die Produktionszyklen mit.

Dieses intensive Wechselverhältnis landwirtschaftlicher Strukturen und industrieller Produktion bei PCAC war von der geografischen Lage des Unternehmens in der Nähe der entsprechenden Rohstoffvorkommen ebenso bedingt wie von dem latenten Personalmangel in den ländlichen Teilen Frankreichs. ${ }^{\text {sI }}$ Diese Rahmenbedingungen stellten für das Unternehmen nicht nur einen Zwang dar, sondern wurden durchaus in das Betriebskalkül der Unternehmensleitung eingepasst. So schrieb ein externer Beobachter 19I3:

"[Die Arbeiter] sind Bauern aus der Gegend, die für acht Stunden zur Arbeit kommen, und anschließend auf ihren Höfen arbeiten. Das Unternehmen kann dadurch auf einen billigen Arbeiterstamm zurückgreifen. [...] Zusätzlich sind sie alle motiviert. Das Unternehmen hat niemals unter Streiks zu leiden gehabt, obwohl doch das nahe gelegene Alais und die Gruben des Gardon zu solchen Konflikten anregen würden. $)^{\text {s2 }}$

Der gleiche Autor machte aber auch die Beobachtung, dass die soziale Struktur der Arbeiterschaft und der ländliche Produktionsraum des Unternehmens ineinandergriffen und eine spezielle Form der Arbeitsorganisationen entstehen ließen. Aufgrund der teilweise enormen Ausdehnung der Produktionsorte - besonders sichtbar am Beispiel der Salinen von Salin de Griaud - konnte die Betriebsführung die Arbeiter meist nur unzureichend kontrollieren.

Der Rationalisierung der Produktion waren somit an allen Standorten enge Grenzen gesetzt. Um diesem Problem zu begegnen standen PCAC zwei Wege offen: Zum einen konnte es versuchen, durch eine leistungsabhängige Bezahlung Anreize zu schaffen;

48 Urteil von 1890 , in: Py 00/13/58 I4.

49 Note sur Salindres von 1920 , in: Py $00 / 13 /$ I9553.

so Vollständige Gehaltslisten der Werksbahnabteilung für den Zeitraum zwischen April und August 1909, in: Py 080/12/58 I4.
5I François Caron, Histoire économique de la France, XIXe - XXe siècle, Paris ${ }^{2}$ I995, 27. Beschreibung der Entwicklung von Salindres M. Favre, 22.5.1920, in: Py 00/13/19953.

52 Archives Crédit Lyonnais Etude économique et financière $1913,21124$. 


\section{Salzernte in Salin de Giraud}

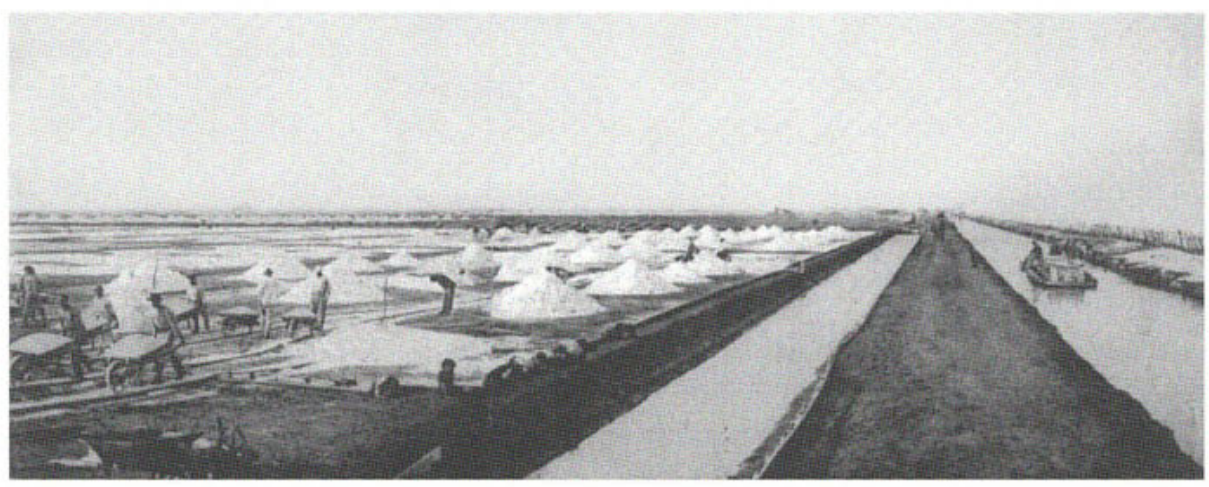

Quelle: Py 00/12/20012.

zum anderen war es aber auch darauf angewiesen, mit möglichst eigenverantwortlichen Arbeitern die Produktion zu organisieren. ${ }^{53}$ Diese Arbeiter mussten zwar keine hohe Qualifikation aufweisen, allerdings mussten sie in der Lage sein, über weite Distanzen zu arbeiten.

In der Tat gibt es Indizien dafür, dass das Unternehmen bestimmte Kompetenzen seiner Arbeiter anders bewertete, als dies etwa bei Bayer die Regel war. Der Arbeitsplatz wurde nicht räumlich eng umrissen, sondern offen gehalten. Besondere topografische Kenntnisse der Region wurden vom Unternehmen honoriert. Die Gratifikationslisten bieten hier Gelegenheit, die Bedeutung dieser Qualifikationen zu skizzieren. Auffällig häufig wurde in den Begründungen für solche Gratifikationen auf Kategorien wie "débrouillard" (gewieft), "débrouillen" (sich durchbeißen), "se tirer d'affaire» (sich zu helfen wissen $)^{54}$ zurückgegriffen, um besondere Qualitäten einzelner Arbeiter zu beschreiben. Diese Charakterisierung an sich scheint auf das hohe Interesse des Unternehmens an möglichst autonom handelnden, aber dennoch loyalen Arbeitern hinzuweisen. Hierzu gesellte sich immer wieder auch der Hinweis auf spezifisch geografische Kontexte. Sowohl in den Salinenbetrieben als auch in den Alpen erschien in den Charakterisierungen der Arbeiter häufig die Orientierungsfähigkeit in der Natur, also jenseits des eigentlichen Werksgeländes, als besonders positives Merkmal. So schlug der Direktor Berne einen seiner Arbeiter mit folgenden Worten für einen Posten als contremaître vor: "er ist sehr robust, energisch und kennt sich in der Gegend sehr gut aus".s5 Die besondere geografische Lage der Produktionsstätten wurde aber auch in Zusammenhang gebracht mit sozialen Problemen, etwa mit der weitgehenden Isolation der Arbeiter in Salin de Giraud. So wurde ein Monsieur Roux dafür honoriert, dass er nicht nur besonders arbeitsam war, sondern darüber hinaus "die besten Verbindungen zu allen

53 Archives Crédit Lyonnais Etude économique et financière 1913,21124 .

54 Alle diese Begriffe stammen aus den Gehaltsvorschlägen von Salin de Giraud von
Direktor Berne an die Verwaltung in Salindres vom 19.4.1909, in: Py 080/12/5814.

s5 Brief von Berne an Salindres vom 28.4.1909, in: Py 080/12/58 I4. 
Werksangehörigen pflegt, eine Sache, die in unserer geografischen Situation von besonderer Bedeutung ist». ${ }^{56}$

Das Unternehmen erkannte, dass die räumliche Ausdehnung nicht nur besondere technische Schwierigkeiten brachte, sondern auch soziale Probleme aufkommen ließ, die man nur durch Anerkennung entsprechender Qualifikationen bei den Arbeitern auffangen konnte. Dies ist umso bemerkenswerter, als die ursprüngliche Personalpolitik von Pechiney zwölf Jahre zuvor noch darauf beruht hatte, gerade diese Salinenarbeiter auf dem Werksgelände einzusperren. ${ }^{57}$ Der Raum wurde bei PCAC nicht im gleichen Maße zum Instrument der Unternehmensplanung, wie dies bei Bayer der Fall war. Doch gerade die Tatsache, dass er für das Unternehmen nicht vollständig einsehbar war, machte ihn zu einer entscheidenden Kategorie in der Produktionskonzeption, die ihren Niederschlag sowohl in der technischen, kommunikativen als auch sozialen Organisation des Unternehmens fand.

\section{Zusammenfassung}

Räumliche Entfernungen wurden bei den besprochenen Unternehmen zunächst als Problem wahrgenommen. Durch Umstrukturierung, Integration und Umsiedlung wurde zwischen den verschiedenen Unternehmensteilen Distanz hergestellt, die Kontroll- und Koordinationsprobleme verursachte. Hinter der Kommunikation zwischen den verschiedenen Unternehmensteilen, verbargen sich offene Konflikte zwischen Prinzipalen und Agenten. Bei Bayer zeigte sich hier der Wille, das Unternehmen weiterhin zentral zu verwalten. Dabei trat in erster Linie Carl Duisberg hervor, der durch das Verfassen seiner Denkschrift nicht nur die Kontrolle der Zentralverwaltung zu stärken suchte, sondern auch seine eigenen Lenkungsambitionen im Unternehmen unterstrich. Unter seiner späteren Führung wurde die Zentralisierung des Unternehmens trotz der unablässigen Expansion weiter vorangetrieben.

Während Bayer versuchte, der Tendenz zur Dezentralisierung durch die Entwicklung einer klaren und kohärenten Raumvorstellung entgegenzutreten, war PCAC in technischer Hinsicht zu einer solchen planmäßigen Erfassung seines Werksgeländes kaum in der Lage. Vielmehr stützte es sich auf technische Notwendigkeiten und vorhandene Strukturen und integrierte diese so weit wie möglich in den organisatorischen Aufbau seines Unternehmens. Hieraus folgte allerdings auch, dass das Unternehmen Qualifikationen seiner Mitarbeiter anders bewertete und ein autonomes, räumliches Wissen zu einer Schlüsselqualifikation seiner Belegschaft machte. Dieses räumliche Wissen gewann auch für die zunehmend komplexen technischen Produktionsabläufe an Bedeutung.

Die hier betrachteten zwei Fallstudien können keine Repräsentativität für die chemische Branche insgesamt in Anspruch nehmen. Es lassen sich anhand dieser Einzelstudien schon gar nicht nationale Modelle entwickeln, die räumliche Ordnungsvorstel-

56 Py $080 / 12 / 5814$.

57 Am 5.10.1897 beschrieb Pechiney dieses Prinzip in einem Bericht vor dem Verwaltungsrat der Firma, in: Py 072/13/29960. Danach habe sich hieraus eine wesentlich stabilere soziale Situation in Salin de Giraud ergeben. Diese Situation war aber nicht von langer Dauer, denn in den nächsten Jahren wurden gerade diese Produktionstätten zu den konfliktreichsten des Unternehmens. 
lungen zeigen würden. Hierfür wäre es nicht zuletzt nötig, die vielfältigen Formen staatlicher und politischer Einflüsse auf den Bereich des Unternehmens zu untersuchen. Gerade die Sicherheits- und Hygienegesetzgebung, die in Deutschland mit der Einführung der Sozialversicherung und in Frankreich mit den Arbeitszeitgesetzen ab den I 890er Jahren vermehrt eine Rolle spielten, ${ }^{8}{ }^{8}$ beeinflussten die unternehmerischen Freiheiten in der Gestaltung der Werksräume. Dennoch erlauben die Untersuchungsergebnisse, Organisations-, Koordinations- und Kontrollmodelle von chemischen Unternehmen darzustellen und adäquat zu charakterisieren. Räumliche Kategorien werden somit zum anschaulichen Begegnungsort zwischen Entwurf und sozial eingebetteter Handlung, die sich mit den persönlichen Ambitionen und Vorstellungen der Akteure verknüpft. So gibt eine solche Kategorie Einblick in unternehmensinterne Dispositionen der Akteure.

Die Unternehmen der Chemiebranche verfügten einerseits über eine relativ große Freiheit in der Gestaltung ihrer Werksflächen. Andererseits standen sie mehr als viele andere Unternehmen im wechselseitigen Verhältnis mit ihrer Umwelt. Hieraus entwickelte sich eine besondere Dynamik in der Reflexion des Raumes, die einige chemische Großunternehmen zu Schlüsselvektoren in der Planung und Nutzung der räumlichen Produktionsdimensionen werden ließen. Ihre Geschichte ist damit auch von besonderer Bedeutung für die Entwicklung der Managementschulen, die den Produktionsraum erst in den $20 \mathrm{er}$ Jahren als eigene Kategorie der Organisationsgestaltung entdeckten. ${ }^{59}$

Adresse des Autors: Dr. des. Heinrich Hartmann, Frankreichzentrum der Freien Universität Berlin, Rheinbabenallee 49, I4I99 Berlin, Email: heinrich.hartmann@fu-berlin.de.

58 Sabine Rudischauser, Vertrag, Tarif, Gesetz. Der politische Liberalismus und die Anfange des Arbeitsrechtes in Frankreich 1890-1902, Berlin I 999; François Guedj/Gérard Vindt, Le temps de travail, une histoire conflictuelle, Paris 1997.

59 Der Fordismus war durch seine Festlegung des Arbeiters auf einen genauen Patz, an dem er zu arbeiten hatte, wohl das erste System, das eine räumliche Festschreibung des Arbeitsplatzes vornahm; Friedrich v. GottlOttilienfeld, Paraphrasen über das Verhältnis von Wirtschaft und technischer Vernunft bei Henry Ford und Frederick W. Taylor, Jena 1924; Chanlat, Space (wie Anm. 30), hier 22. 